\title{
Erratum to: Quantitative proteomics analysis reveals that $S$-nitrosoglutathione reductase (GSNOR) and nitric oxide signaling enhance poplar defense against chilling stress
}

\author{
Tielong Cheng ${ }^{1} \cdot$ Jinhui Chen ${ }^{1} \cdot$ Abd_Allah $\mathrm{EF}^{2} \cdot$ Pengkai Wang ${ }^{1} \cdot$ \\ Guangping Wang ${ }^{1} \cdot$ Xiangyang $\mathrm{Hu}^{3} \cdot \mathrm{Jisen} \mathrm{Shi}^{1}$
}

Published online: 15 March 2016

(C) Springer-Verlag Berlin Heidelberg 2016

\section{Erratum to: Planta (2015) 242:1361-1390 DOI 10.1007/s00425-015-2374-5}

The author would like to correct the error in the publication of the original article.

The Acknowledgments section should read as:

This work was supported by the National High Technology Research and Development Program of China (863 Program, No. 2013AA102705), the National Key Basic Research Program of China (No. 2012CB114500), the
National Science Foundation of China (No. 31170619), the Program for New Century Excellent Talents by the Ministry of Education, the Talent Project by Ministry of Science and the Technology and Priority Academic Program Development of Jiangsu Higher Education Institutions. The authors would like to extend their sincere appreciation to the Deanship of Scientific Research at king Saud University for its funding this Research group (No. RG 1435-014).
The online version of the original article can be found under doi:10.1007/s00425-015-2374-5.

Jisen Shi

jshi@njfu.edu.cn

Xiangyang $\mathrm{Hu}$

huxiangyang@mail.kib.ac.cn

1 Key Laboratory of Forest Genetics \& Biogeography, Ministry of Education, Nanjing Forest University, Nanjing 210037, China

2 Department of Plant Production, Faculty of Food \& Agricultural Sciences, King Saud University, Riyadh, Saudi Arabia

3 Key Laboratory for Plant Diversity and Biogeography of East Asia, Kunming Institute of Botany, Chinese Academy of Science, Kunming 650201, China 